Accretion Phenomena and Related Outflows, IAU Colloquium 163

ASP Conference Series, Vol. 121, 1997

D.T. Wickramasinghe, L. Ferrario, and G.V. Bicknell, eds.

\title{
Accretion in the Center of the Milky Way
}

\author{
Wolfgang J. Duschl \\ Institut für Theoretische Astrophysik, Heidelberg, Germany \\ Max-Planck-Institut für Radioastronomie, Bonn, Germany \\ Susanne von Linden \\ Landessternwarte, Heidelberg, Germany
}

\begin{abstract}
We have analyzed the distribution of giant molecular clouds and their radial velocities in the inner $\sim 300 \mathrm{pc}$ of the Milky Way in order to deduce the characteristic properties of its large scale motion. We find radial inflow of matter towards the Galactic Center of $\sim 10^{-2} \mathrm{M}_{\odot} / \mathrm{yr}$. Moreover, we have determined the position of individual clouds along the line of sight.
\end{abstract}

\section{Introduction}

Gas and dust in the inner parts of the Galaxy are concentrated mainly in a disk-like distribution. Several surveys have determined details of the density distribution and of the radial velocities (for a detailled summary, see Mezger, Duschl and Zylka 1996).

We model the dynamics of the molecular cloud distribution in the inner $\sim 200 \mathrm{pc}$ from the Galactic Center (GC) in the framework of an accretion disk. The two parameters that mainly determine the structure of such a disk are the radial mass flow rate through the disk $(\dot{M})$ and the dynamic viscosity $(\nu)$. For any pair of these two parameters, one can calculate the radial velocities $\left(v_{s}\right)$ in the disk with respect to the $\mathrm{GC}$ and, consequently, the observable quantity $v_{x}$, the radial velocity with respect to the observer (of course, after applying all the relevant transformations). In an iterative process, with this method, we determine the pair $\{\dot{M}, \nu\}$ that gives the best agreement between the observed and modelled values of $v_{x}$. Having found such a solution, we also determined the location of the individual clouds with respect to the GC. Thus, this technique allows us also to construct a map of the distribution of molecular clouds close to the GC. In the next Section, we summarize our ansatz for describing the accretion disk. In Section 3, we demonstrate our technique for one example, and in Section 4, we summarize the results and compare them to parameters that have been determined independently. Finally, the last Section is devoted to an overview of the process that allows for the physical understanding of the determined quantities. 


\section{The accretion disk model}

We describe the dynamics of the molecular clouds in the vicinity of the GC in the framework of an accretion disk model. For this, we assume that the molecular clouds are the only prominent features in an otherwise smooth disk flow. This allows us to use the observed radial velocities of the cloud as characteristic for the whole disk at the galactocentric radius of the cloud, and thus to use the clouds as tracers of the gas flow in the disk.

In the following, we only discuss the case of stationary accretion disks with no mass input other than the radial one at the disk's outer edge. We model the disk as being geometrically thin and azimuthally symmetric. The disk is assumed to reside in a spherically symmetric potential that is due to a prescribed mass distribution $\left(M_{\mathrm{b}}(r)\right)$. In the case of the $\mathrm{GC}$, we take $M_{\mathrm{b}}(r)=1.14$. $10^{9} M_{\odot} \cdot(r / 100 \mathrm{pc})^{5 / 4}(r$ is the radius in a spherical coordinate system) (see, e.g., Genzel and Townes 1987). This defines the azimuthal velocity, i.e., the Keplerian velocity $\left(v_{\phi}\right)$.

The dynamical viscosity is assumed to follow a power law in radial direction: $\nu=\nu_{0} \cdot\left(s / s_{0}\right)^{\beta} . s$ is the radius in a cylindrical coordinate system which is the appropriate choice for a geometrically thin accretion system. Only in the plane of symmetry is $s=r . s_{0}$ is an arbitrarily chosen scaling radius.

\section{M-0.13-0.08 - An example}

As an example, we have chosen one of the best observed molecular clouds in the GC area, namely M-0.13-0.08 and its vicinity. For validation of the method, we have taken a comparatively small set of seven observations. The observations are taken from the large sample of Zylka, Mezger, and Wink (1990). By matching observed and theoretically modelled radial velocities $v_{x}$, we get as the best fit pair of parameters $\dot{M}=10^{-1.8} M_{\odot} / \mathrm{yr}$, and $\nu(s=115 \mathrm{pc})=6 \cdot 10^{26} \mathrm{~cm}^{2} / \mathrm{sec}$. The radius of $115 \mathrm{pc}$ is the average radius from the GC of the seven observations. This is thus the determined radial distance of M-0.13-0.08 from the GC.

\section{The parameters of the disk}

Obviously, the determination of one cloud position in the GC accretion disk does not allow us to determine radial variations of the viscosity. On the other hand, the determined radius of $\sim 115 \mathrm{pc}$ from the GC is well within the limits of $30 \ldots 200 \mathrm{pc}$ inferred from spectroscopy $(s>30 \mathrm{pc})$ and dynamics of the expanding molecular ring $(s<200 \mathrm{pc})$.

To get additional information about the radial variations of $\nu$, we choose to include observations of Pauls et al. (1993) of material presumably much closer to the GC. We find that, indeed, this material is located at a GC distance of only $\sim 15$ pc. Keeping the assumption of a stationary disk (i.e., $\dot{M}=$ const.), this allows us to determine the radial derivative of $\nu$. We find $\nu(s)=5.7$. $10^{26} \mathrm{~cm}^{2} / \mathrm{sec} \cdot(s / 100 \mathrm{pc})^{0.4}$.

For the above mentioned radial mass flow rate, together with this viscosity, we have to choose the disk's inner radius at $\ll 15$ pc to ensure a) that numerical 
effects of the boundary condition do not influence the solution, and b) that the basic assumptions of the disk models (e.g., $v_{s} \ll v_{\phi}$ ) are still valid at the location of the clouds. To fulfill both conditions, in our final models, we put the inner disk boundary at $1 \mathrm{pc}$. It is important to note that this choice does not represent a physically mandated radius but one that guarantees numerical consistencies at the locations where the analysed structures are located.

It is also important to note that the viscosity that we have determined in this way is in good agreement with what one can independently estimate to be a reasonable value. Interpreting it as some kind of turbulent viscosity, we may estimate its value by taking the product of the observed velocity dispersion in the gas and the scale height of the gas distribution. In the case of the GC this, interestingly enough, leads to a number that agrees to within an order of magnitude with the one that we have determined through matching the radial velocities.

\section{A mechanism generating high viscosities}

Despite the fact that we find agreement between the characteristic velocities and lengths scales as observed in the GC, and the viscosity that our model calculations indicate, this kind of analysis does not provide a physical explanation of the viscosity.

Assuming a standard $\alpha$ accretion disk (Shakura and Sunyeav 1973) would immediately lead to a viscosity parameter $\alpha$ that is by several orders of magnitude larger than the usually assumed maximum value of 1 . This maximum applies only to the case of a closed system, i.e., to an accretion disk that has its only connection to the outside through its radiation.

If we allow for other processes, like interactions with bars or a stellar component, the classical $\alpha$ prescription breaks down. On the other hand, one can think of several other processes in the GC environment that would be capable of putting energy into the disk and thus would make (much) higher viscosities possible.

Linden et al. (1993) have proposed such a process and have shown that supernovae could very well put enough energy into the accretion disk to allow for such a high viscosity. Linden et al. have even succeeded in showing that there is a cyclic evolution possible that can maintain itself. Supernovae gives rise to a high enough energy input to explain the observed viscosity. The high viscosity, in turn, causes such large a mass flow rate that there is always sufficient mass replenished to ensure ongoing star formation and thus to ensure a high enough supernovae rate.

In a forthcoming paper, Duschl, Strittmatter and Biermann (1997) discuss a generalization of the $\alpha$ ansatz that allows also for considerably larger viscosities than in the classical prescription, especially if self-gravity of the disk plays a role.

Acknowledgments. We benefitted much from discussions with P.L. Biermann. We thank R. Auer for his comments on the manuscript. This work is being supported by the Deutsche Forschungsgemeinschaft through grant $S F B$ 328. 


\section{References}

Duschl W.J., Strittmatter P.A., Biermann P.L., 1997, ApJ, submitted Genzel, R., Townes, C.H. 1987, ARAA, 25, 377

Linden, S.v., Biermann, P.L., Duschl, W.J., Lesch, H., Schmutzler, T., 1993, A\&A, 280, 468

Mezger P.G., Duschl W.J., Zylka R., 1996, AAR, in press

Pauls, T., et al. 1993, ApJ, 403, L13

Shakura, N.I. and Sunyaev, R.A. 1973, A\&A, 24, 337

Zylka, R., Mezger, P.G., Wink J.E. 1990, A\&A, 234, 133

\section{Discussion}

R. Sunyaev: Recent discovery by ASCA of strong iron $\mathrm{K} \alpha$-line $(6.9 \mathrm{keV})$ emission from SGR B2 molecular complex might be interpreted as a result of irradiation by the Sgr $A^{*}$ flare which occurred several hundreds of years ago with $\mathrm{X}$-Ray luminosity above $10^{40} \mathrm{ergs} \mathrm{s}^{-1}$. What is your opinion - could such a flare heat the gas within $3 \mathrm{pc}$, form a hole in the gas distribution, and lead to the low accretion rate at the present time?

W. Duschl: We are working on this, but we do not have final results yet. From first calculations it seems as if the timescale of $\sim 10^{2}$ years is a little short to "switch off" the accretion in the centre of the galaxy. But we have to wait for the final results to be sure. 